\section{Thromboseprophylaxe bei ambulanten Patienten}

Eine Prophylaxe venöser Thromboembolien (VTE) wird bei ambulanten Onkologiepatienten selten durchgeführt. Es fehlt an genauen Informationen z. B. zu Inzidenz und Risikofaktoren. Eine große Kohortenstudie liefert Anhaltspunkte.

$\mathrm{D}$ atenbasis der retrospektiven Kohortenstudie war die IMS/PharMetrics Patient-Centric database im Zeitraum zwischen Januar 2004 bis Dezember 2009. Extrahiert wurden demographische und klinische Charakteristika der Krebspatientenkohorte $(\mathrm{n}=17.284)$ und diese Daten individuell im Verhältnis 1:1 mit denen einer von Alter- und Geschlechtsverhältnis vergleichbaren Kontrollgruppe mit Nicht-Krebspatienten verglichen. Die Patienten hatten sechs der häufigsten Krebsarten (Lunge, Pankreas, Gastrointestinalsystem, Kolon/ Rektum, Blase, Ovar). Die VTE-Inzidenz wurde über eine drei- bis 12-mona- tige Beobachtungsperiode ermittelt, die nach Beginn der Chemotherapie (CT) begann. In multivariaten Analysen sollten unabhängige Prädiktoren von VTE und Blutungen bei den ambulanten $\mathrm{Pa}$ tienten ermittelt werden.

Das durchschnittliche Alter der Studienpopulation war 64 Jahre, $51 \%$ der Patienten Frauen. In der Krebspatientenkohorte trat eine VTE bei 12,6\% (n $=2.170$ ) innerhalb von 12 Monaten nach Beginn der CT auf im Vergleich $\mathrm{zu} 1,4 \%$ der Kontrollpersonen $(\mathrm{n}=237$, $\mathrm{p}<0,001)$. Die VTE-Inzidenz war $\mathrm{ab}$ hängig von der Entität und variierte von 19,2 (Pankreaskarzinom) bis 8,2\%
(Blasenkarzinom). Als Prädiktoren einer VTE wurden neben der Krebsart außerdem Komorbiditäten sowie häufig verordnete Krebsmedikamente bzw. Supportive Care-Medikamente wie Cisplatin, Bevacizumab und Erythropoietin ermittelt.

Fazit: Bei ambulanten Patienten mit soliden Tumoren ist die Rate an VTE bis zu 12 Monate nach CT-Beginn mit rund $12 \%$ etwa neunmal höher als bei vergleichbaren nicht onkologischen $\mathrm{Pa}$ tienten. Bei erhöhtem Risiko in den Bereichen Krebsentität, Komorbiditäten und verabreichte Medikamente sollte eine gezielte Thromboembolie-Prophylaxe eingeleitet werden.

Barbara Kreutzkamp

Khorana AA et al. Incidence and predictors of venous thromboembolism (VTE) among ambulatory high-risk cancer patients undergoing chemotherapy in the United States. Cancer. 2013;119(3):648-55.

\section{Knochenmetastasen: Weniger Komplikationen, bessere Lebensqualität}

\section{In einer Phase-III-Studie war der monoklonale Antikörper Denosumab dem Bisphosphonat Zoledronat hinsichtlich der Verhinderung oder Verzögerung von skelettbezogenen Ereignissen nicht unterlegen. Nun liegen auch die Ergebnisse zur Lebensqualität vor.}

- ortschritte in der Krebstherapie haben dazu geführt, dass Patienten mit Knochenmetastasen immer länger leben. Skelettbezogene Ereignisse wie Frakturen, Bestrahlung des Knochens, Operationen am Knochen oder Kompression des Rückenmarks verursachen eine erhöhte Morbidität, Schmerzen und eine Beeinträchtigung der Mobilität. Daher gewinnen Strategien zur Prävention von Komplikationen wie skelettbezogenen Ereignissen und Schmerzen zunehmend an Bedeutung.

An der Phase-III-Studie, in der der Nutzen von Denosumab und Zoledronat im Hinblick auf skelettbezogene Ereignisse verglichen wurde, nahmen 1.776 Patienten mit fortgeschrittener Krebserkrankung (ausgenommen Brust- und Prostatakrebs) oder multiplem Myelom teil. Die Behandlung erfolgte randomi- siert entweder mit Denosumab (120 mg s.c., alle vier Wochen) oder Zoledronat (4 mg i.v., alle vier Wochen).

Denosumab verzögerte die Zeit bis zum erstmaligen Auftreten eines skelettbezogenen Ereignisses, einer Hyperkalzämie oder einer Malignität im Vergleich zu Zoledronat (14,4 Monate vs. 19 Monate). Auch waren skelettbezogene Ereignisse insgesamt unter Denosumab seltener als unter Zoledronat (31,4 vs. 36,3\% der Patienten). Eine Bestrahlung der Knochen war unter dem Antikörper um $22 \%$ seltener notwendig als unter dem Bisphosphonat ( $\mathrm{p}=0,026)$.

Die Number-needed-to-treat (NNT), um ein skelettbezogenes Ereignis zu vermeiden, lag unter Denosumab versus Placebo bei drei Patientenjahren und unter Zoledronat bei zehn Patientenjahren.
Auch hinsichtlich der Schmerzen war Denosumab effektiv. Die Substanz verhinderte eine relevante Verschlimmerung von Schmerzen, definiert als ein Anstieg um zwei Punkte im Brief Pain Inventory Score, signifikant um $15 \%$ gegenüber Zoledronat $(\mathrm{p}<0,05)$. Bei Patienten, die zu Studienbeginn keine oder nur leichte Schmerzen hatten, verzögerte Denosumab die Zeit bis zum Auftreten moderater bis schwerer Schmerzen $(p=0,04)$. Auch die Folgen der Schmerzen auf andere Aspekte des Lebens, z. B. Aktivität oder Stimmung, beeinflusste Denosumab positiv. Die gesundheitsbezogene Lebensqualität, beurteilt mithilfe des FACT-G, war in beiden Gruppen vergleichbar.

Fazit: Die Behandlung mit Denosumab schützt Patienten mit soliden Tumoren oder multiplem Myelom besser vor Komplikationen von Knochenmetastasen als Zoledronat. Die Ergebnisse sprechen für eine frühzeitige Behandlung.

Judith Neumaier

Vadhan-Raj S et al. Clinical benefit in patients with metastatic bone disease: results of a phase 3 study of denosumab versus zoledronic acid. Ann Oncol. 2012;23(12):3045-51. 